\title{
A POSSIBLE EXPLANATION OF DISTORTIONS IN THE LIGHT CURVES OF VW CEP BY STARSPOTS
}

\author{
A. Yamasaki \\ Dept. Earth Science and Astronomy, \\ University of Tokyo, Tokyo 153, Japan
}

\section{ABSTRACT}

An attempt is made to explain the variations in the light curve of VW Cep in terms of a starspot model. Based upon Kwee's (1966) observations, an unperturbed light curve without spots is constructed and analysed for the geometrical elements of $\mathrm{VW}$ Cep. Then, by assuming that starspots exist on the surface of the primary component, Kwee's individual light curves are further analysed for locations and intensities of the starspots.

VW Cep $(P=0.278)$ is a well-known $W$ UMa type system showing variations in the light curve and in the orbital period. Several important papers (e.g., Walter, 1979) have been published so far to interpret these variations. In the present paper, it is shown that variations in the light curve will be explained by a starspot hypothesis.

Starspot models for late type variables such as BY Dra stars and RS CVn stars have given successful explanations of distortions in the light and flare-like activities sometimes observed in these stars. Because of the similarities between VW Cep ( $\mathrm{KOV}$ ) and RS CVn stars in the spectral types and in the nature of light curve distortions, it may be worth trying to apply a starspot model to VW Cep.

To determine distributions and intensities of starspots, an unspotted light curve which is free from starspots should be necessary as the reference. However, each observed light curve seems to suffer from some amounts of distortions. Therefore, an unspotted light curve has to be constructed from observed light curves by taking the maximum light among observed light curves for every corresponding phase (no hot spots, no enhancement of the continuum radiation are assumed).

This process has been applied to Kwee's (1966) observations which were made between 1957 and 1959 at $\lambda 5600$, to obtain the unspotted light curve. Then, the unspotted light curve has been analysed by the light- 
curve synthesis method for the geometrical elements.

For the mass ratio $q=0.41$ (Binnendijk, 1967), the elements

$i=64^{\circ} \pm 1^{\circ}$

$f=1.10 \pm 0.05$

$j=1.82 \pm 0.05$

have been obtained, where $f$ and $j$ denote the fill-out-ratio and the ratio of the surface brightness (Yamasaki, 1981), respectively, while for the mass ratio $q=0.33$ (Popper, 1948), the slightly different element

$$
\begin{aligned}
& i=65^{\circ} \pm 1^{\circ} \\
& f=1.10 \pm 0.05 \\
& j=1.77 \pm 0.05
\end{aligned}
$$

have been obtained. Note that adopting the effective temperature for the primary component $\mathrm{T}_{\text {effl }}=5300 \mathrm{~K}$ for $\mathrm{KOV}$, we obtain that the temperature for the secondary component can be estimated to be around $T_{\text {eff } 2}=6100 \mathrm{~K}$, hence the system reveals a rather large temperature difference.?

Then, according to Mullan (1975), starspots on the surface of the primary component have been considered. Four light curves, on JD2436124 (epoch I), JD2436285 (epoch II), JD2436448 (epoch III) and JD2436679 (epoch IV), among Kwee's (1966) observations have been used to find out positions and intensities of starspots.

The surface of the primary component has been divided into 66 regions where $S, B, P$ and $P^{\prime}$ represent $L_{1}$ point $\left(\lambda=0^{\circ}, \phi=0^{\circ}\right)$, the back point $\left(\lambda=180^{\circ}, \phi=0^{\circ}\right)$, the pole visible from the Earth $\left(\phi=90^{\circ}\right)$ and the pole invisible from the Earth $\left(\phi=-90^{\circ}\right)$, respectively. Phases $0^{\circ}, 90^{\circ}$, $180^{\circ}$ and $270^{\circ}$ correspond to the transit minimum (the photometric secondary minimum), the second maximum (points $\lambda=90^{\circ}$ face the Earth), the occultation minimum (the photometric primary minimum) and the first maximum (points $\lambda=270^{\circ}$ face the Earth), respectively.

Figure 1 shows Kwee's observations, the starspot model solutions (solid line) and the distribution of starspots (the intensities of starspots are represented by the darkening of the shades).

The movement of starspots can be clearly seen in Figure 1, where starspots are gathered around $\lambda=0^{\circ}, 270^{\circ}, 180^{\circ}$ and $90^{\circ}$ for epochs $\mathrm{I}$, II, III and IV, respectively. This may indicate that starspots rotate on the surface of the primary component a little faster (by 0.048) than the synchronized rotation.

Recent discovery of X-ray emission from Vw Cep (Carroll et al., 1980) and the intense UV emissions (Dupree et al., 1979) would suggest activities which are associated with starspots on the surface of VW Cep. 

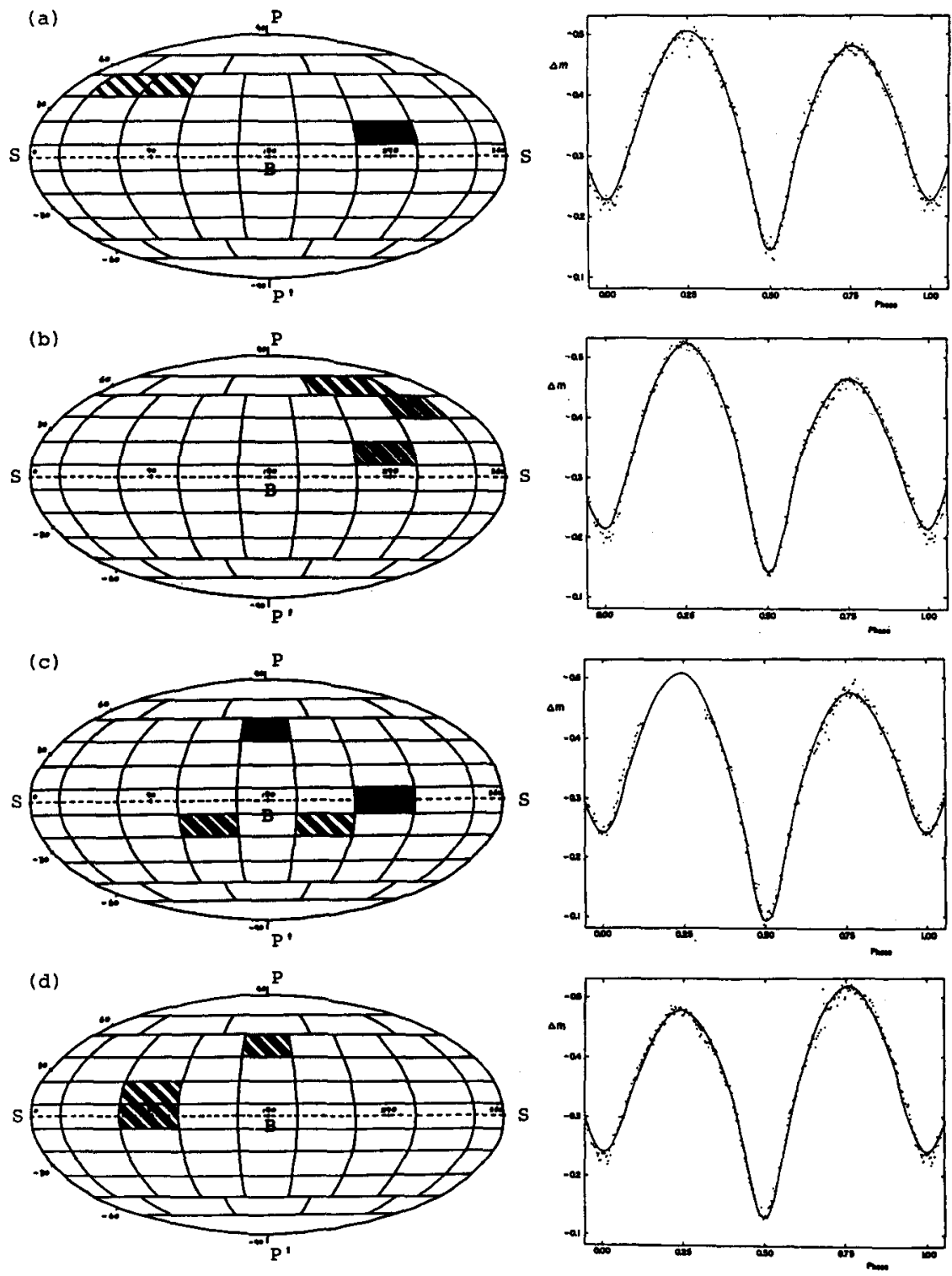

Figure 1. Individual observations (Kwee, 1966), the starspot model solutions (solid line) and the distribution of starspots on the surface of the primary component of VW Cep for (a) epoch I (JD2436124), (b) epoch II (JD 2436285), (c) epoch III (JD2436448) and (d) epoch IV (JD2436679), respectively. 
This work was supported in part by the Scientific Research Fund of the Ministry of Education, Science and Culture (56540137).

\section{REFERENCES}

Binnendijk, L.: 1967, Publ. Dominion Astrophys. Obs. 13, 27.

Carroll, R.W., Cruddace, R.G., Friedman, H., Byram, E.T., Wood, K., Meekins, J., Yentis, D., Share, G.H., and Chubb, T.A.: 1980, Astrophys. J. 235, L77.

Dupree, A.K., Black, J.H., Davis, R.J., Hartmann, I., and Raymond, J.C.: 1979, The First Year of IUE, University College London.

Kwee, K.K.: 1966, Bull. Astron. Inst. Netherlands Supp1. 1, 245.

Mullan, D.J.: 1975, Astrophys. J. 198, 563.

Popper, D.M.: 1948, Astrophys. J. 108, 490.

Walter, K.: 1979, Astron. Astrophys. 80, 27.

Yamasaki, A.: 1981, Astrophys. Space Sci. 77, 75. 